\title{
Numerical Solution of Uncertain Beam Equations Using Double Parametric Form of Fuzzy Numbers
}

\author{
Smita Tapaswini and S. Chakraverty \\ Department of Mathematics, National Institute of Technology, Rourkela, Odisha 769 008, India \\ Correspondence should be addressed to S. Chakraverty; sne_chak@yahoo.com
}

Received 31 March 2013; Revised 17 June 2013; Accepted 11 September 2013

Academic Editor: Christian W. Dawson

Copyright ( 12013 S. Tapaswini and S. Chakraverty. This is an open access article distributed under the Creative Commons Attribution License, which permits unrestricted use, distribution, and reproduction in any medium, provided the original work is properly cited.

\begin{abstract}
Present paper proposes a new technique to solve uncertain beam equation using double parametric form of fuzzy numbers. Uncertainties appearing in the initial conditions are taken in terms of triangular fuzzy number. Using the single parametric form, the fuzzy beam equation is converted first to an interval-based fuzzy differential equation. Next, this differential equation is transformed to crisp form by applying double parametric form of fuzzy number. Finally, the same is solved by homotopy perturbation method (HPM) to obtain the uncertain responses subject to unit step and impulse loads. Obtained results are depicted in terms of plots to show the efficiency and powerfulness of the methodology.
\end{abstract}

\section{Introduction}

Recently, theory of differential equations plays a vital role to model physical and engineering problems such as in solid and fluid mechanics, viscoelasticity, biology, physics, and other areas of science. But in actual case, the parameters, variables, and initial conditions involved in the differential equations may be uncertain, or a vague estimation of those are found in general by some observation, experiment, experience, or maintenance induced error. So, to overcome the uncertainty and vagueness, one may use fuzzy environment in parameters, variables, and initial condition in place of crisp (fixed) ones. So, with these uncertainties the general differential equations turn into fuzzy differential equations (FDEs). In real-life application, it is too complicated to obtain the exact solution to fuzzy differential equations, so one may need a reliable and efficient numerical technique for the solution for fuzzy differential equations.

There exist a large number of papers dealing with fuzzy differential equations and its applications in the open literature. Some are reviewed and cited here for better understanding of the present analysis. Chang and Zadeh [1] first introduced the concept of a fuzzy derivative, followed by Dubois and Prade [2] who defined and used the extension principle in their approach. The fuzzy differential equations and fuzzy initial value problems are studied by Kaleva $[3,4]$ and Seikkala [5]. Various numerical methods for solving fuzzy differential equations are introduced in [6-16]. Ma et al. [6] developed a scheme based on the classical Euler method to solve fuzzy ordinary differential equations. A two-dimensional differential transform method to solve fuzzy partial differential equations (FPDEs) has been studied in [7]. Abbasbandy and Allahviranloo [8] applied Taylor method for the solutions of fuzzy differential equations. Abbasbandy et al. [9] developed a numerical method for solving fuzzy differential inclusions, and in the proposed method, fuzzy reachable set is used to approximate the solution. Variation of constant formula is handled by Khastan et al. [10] to solve first-order fuzzy differential equations. Variational iteration method is discussed by Allahviranloo et al. [11] to obtain the exact solutions of fuzzy wave-like equations with variable coefficients. The concept of generalized $\mathrm{H}$-differentiability is studied by Chalco-Cano and Román-Flores [12] to solve fuzzy differential equations. Mondal and Roy [13] described the solution procedure of a first-order linear non homogeneous ordinary differential equation in fuzzy environment. Palligkinis et al. [14] applied the Runge-Kutta method for more general problems and proved the convergence for s-stage Runge-Kutta methods. Akin et al. [15] developed an algorithm based on $\alpha$ cut of a fuzzy set for the solution of second-order fuzzy initial 
value problems. Very recently, Khastan et al. [10] proposed improved Euler method for the solution of fuzzy differential equations.

Similarly, many authors studied various other methods to solve $n$th order fuzzy differential equations in [17-21]. Based on the idea of collocation method, Allahviranloo et al. [17] investigated the numerical solution of $n$th order fuzzy differential equations. Jafari et al. [18] used variational iteration method for solving $n$ th-order fuzzy differential equations recently. Parandin [19] discussed Runge-Kutta method for the numerical solution of fuzzy differential equations of $n$ thorder. Hashemi et al. [20] studied homotopy analysis method for the solution of system of fuzzy differential equations. Zhang and Wang [21] utilized time domain methods for the solutions of $n$ th-order fuzzy differential equations.

Recently, homotopy perturbation method is found to be a powerful tool for linear and nonlinear differential equations. The HPM was first developed by He in [22, 23], and many authors applied this method to solve various linear and nonlinear functional equations of scientific and engineering problems $[24,25]$. The solution is considered as the sum of infinite series, which converges rapidly to accurate solutions. In the homotopy technique (in topology), a homotopy is constructed with an embedding parameter $p \in[0,1]$, which is considered as a "small parameter". Very recently homotopy perturbation method has been implemented to a wide class of problems. Few researchers have also investigated the solution of fuzzy differential equations using HPM. Allahviranloo and Hashemzehi [26] successfully applied homotopy perturbation method for the solution of fuzzy-fredholm integral equations. Babolian et al. [27] solved integro-differential equations using homotopy perturbation method. Matinfar and Saeidy [28] handled an application of homotopy perturbation method for fuzzy integral equations. Numerical solution of fuzzy initial value problems under generalized differentiability by HPM is studied by Ghanbari [29]. Recently, Tapaswini and Chakraverty [30] implemented HPM for the solution of $n$ th-order fuzzy linear differential equations.

Moreover, Bede [31] described the exact solutions of fuzzy differential equations in his note in an excellent way. Ahmada et al. [32] study analytical and numerical solutions of fuzzy differential equations based on the extension principle. Buckley and Feuring [33] applied two analytical methods for solving $n$ th-order linear differential equations with fuzzy initial conditions. In the first method, they simply fuzzify the crisp solution to obtain a fuzzy function and then check whether it satisfies the differential equation or not. And the second method was just the reverse of the first method.

The above literature review reveals that the fuzzy differential equations are always converted to two crisp differential equations. Then corresponding crisp systems are solved to obtain the bounds of fuzzy solution. But here, a new method is proposed based on double parametric form of fuzzy as define in Definition 2 where fuzzy differential equation has been converted to a single crisp differential equation. Finally the corresponding crisp (single) differential equation is solved by homotopy perturbation method to obtain the fuzzy solution in double parametric form.
This paper is organized as follows. In Section 2, some basic preliminaries related to the present investigation are given. Section 3 represents the basic idea of HPM. Proposed technique is applied with HPM in Section 4 to solve uncertain beam equation. Next, uncertain response analysis is presented followed by numerical results and discussions. Finally, in Section 7 conclusions are drawn.

\section{Preliminaries}

In this section, we present some notations, definitions, and preliminaries which are used further in this paper.

Definition 1 (single parametric form of fuzzy numbers). The triangular fuzzy number $\widetilde{U}=(a, b, c)$ can be represented with an ordered pair of functions through $\alpha$-cut approach; namely, $[\underline{u}(\alpha), \bar{u}(\alpha)]=[(b-a) \alpha+a,-(c-b) \alpha+c]$ where, $\alpha \in[0,1]$.

The $\alpha$-cut form is known as parametric form or single parametric form of fuzzy numbers.

It may be noted that the lower and upper bounds of the fuzzy numbers satisfy the following requirements.

(i) $\underline{u}(\alpha)$ is a bounded left continuous nondecreasing function over $[0,1]$.

(ii) $\bar{u}(\alpha)$ is a bounded right continuous nonincreasing function over $[0,1]$.

(iii) $\underline{u}(\alpha) \leq \bar{u}(\alpha), 0 \leq \alpha \leq 1$.

Definition 2 (double parametric form of fuzzy number). Using the parametric form as discussed in Definition 1 one has $\widetilde{U}=[\underline{u}(\alpha), \bar{u}(\alpha)]$. Now one may write this as crisp number with double parametric form as $\widetilde{U}(\alpha, \beta)=\beta(\overline{\mathcal{u}}(\alpha)-\underline{u}(\alpha))+$ $\underline{u}(\alpha)$, where $\alpha$ and $\beta \in[0,1]$.

\section{Homotopy Perturbation Method $[22,23]$}

To illustrate the basic idea of this method, we consider the following nonlinear differential equation of the form:

$$
A(u)-f(r)=0, \quad r \in \Omega,
$$

with the boundary condition

$$
B\left(u, \frac{\partial u}{\partial n}\right)=0, \quad r \in \Gamma,
$$

where $A$ is a general differential operator, $B$ a boundary operator, $f(r)$ a known analytical function, and $\Gamma$ is the boundary of the domain $\Omega$. A can be divided into two parts which are $L$ and $N$, where $L$ is linear and $N$ is nonlinear. Equation (1) can therefore be written as follows:

$$
L(u)+N(u)-f(r)=0, \quad r \in \Omega .
$$

By the homotopy technique, we construct a homotopy $U(r, p): \Omega \times[0,1] \rightarrow R$, which satisfies

$$
\begin{aligned}
H(U, p) & =(1-p)\left[L(U)-L\left(v_{0}\right)\right]+p[A(U)-f(r)] \\
& =0, \quad p \in[0,1], r \in \Omega
\end{aligned}
$$


or

$$
H(U, p)=L(U)-L\left(v_{0}\right)+p L\left(v_{0}\right)+p[N(U)-f(r)]=0,
$$

where $r \in \Omega, p \in[0,1]$ is an embedding parameter, and $v_{0}$ is an initial approximation of (1). Hence, it is obvious that

$$
\begin{aligned}
& H(U, 0)=L(U)-L\left(v_{0}\right)=0, \\
& H(U, 1)=A(U)-f(r)=0,
\end{aligned}
$$

and the changing process of $p$ from 0 to 1 is just that of $H(U, p)$ from $L(U)-L\left(v_{0}\right)$ to $A(U)-f(r)$. In topology, this is called deformation, $L(U)-L\left(v_{0}\right)$ and $A(U)-f(r)$ is called homotopic. Applying the perturbation technique [22, 23], due to the fact that $0 \leq p \leq 1$ can be considered as a small parameter, we can assume that the solution of (4) or (5) can be expressed as a series in $p$ as follows:

$$
U=u_{0}+p u_{1}+p^{2} u_{2}+p^{3} u_{3}+\cdots
$$

when $p \rightarrow 1$, (4) or (5) corresponds to (3); Then (7) becomes the approximate solution of (3), that is,

$$
u=\lim _{p \rightarrow 1} U=u_{0}+u_{1}+u_{2}+u_{3}+\cdots
$$

The convergence of the series (8) has been proved in $[22,23]$.

\section{Proposed Method}

Here, we first convert the fuzzy differential equation to interval-based fuzzy differential equation using single parametric form. Then by using double parametric form, interval-based fuzzy differential equation is reduced to crisp differential equation. Next, we apply homotopy perturbation method to solve the corresponding differential equation. As per the titled problem, let us now consider the fuzzy beam equation

$$
\rho A \frac{\partial^{2} \widetilde{v}}{\partial t^{2}}+c \frac{\partial \widetilde{v}}{\partial t}+E I \frac{\partial^{4} \widetilde{v}}{\partial x^{4}}=F(x, t)
$$

This may be written as

$$
\frac{\partial^{2} \widetilde{v}}{\partial t^{2}}+\frac{c}{\rho A} \frac{\partial \widetilde{v}}{\partial t}+\frac{E I}{\rho A} \frac{\partial^{4} \widetilde{v}}{\partial x^{4}}=\frac{F(x, t)}{\rho A},
$$

where $\rho, A, c, E$, and $I$ represent the mass, density, cross sectional area, damping coefficients per unit length, Young's modulus of elasticity, and moment of inertia of the beam. $F(x, t)$ is the externally applied force and $\widetilde{v}(x, t ; \alpha)$ is the transverse fuzzy displacement. Fuzzy initial conditions are considered as $\widetilde{v}(0)=\widetilde{v}^{\prime}(0)=(-0.1,0,0.1)$. It may be noted that if the initial condition is crisp, then we may have the initial condition as $v(0)=v^{\prime}(0)=0$. Here, the initial condition has been taken as fuzzy with an idea that the condition may actually be uncertain; namely, it may be due to error in observation or experiment and so forth, where we may take the error or uncertainty as left and right spread as -0.1 and 0.1 , respectively in terms of fuzzy triangular membership function. As such this will force the governing differential equation as a whole as uncertain. So naturally, the outcome or the output (result) must be uncertain. This way we may have the actual essence of the uncertainty in response which may benefit the engineers to understand the safety of the system in a better way. So, we need to have efficient methods to handle these problems.

As per the single parametric form we may write the above fuzzy differential equation (10) as

$$
\begin{gathered}
{\left[\frac{\partial^{2} \underline{v}(t ; \alpha)}{\partial t^{2}}, \frac{\partial^{2} \bar{v}(t ; \alpha)}{\partial t^{2}}\right]+\frac{c}{\rho A}\left[\frac{\partial \underline{v}(t ; \alpha)}{\partial t}, \frac{\partial \bar{v}(t ; \alpha)}{\partial t}\right]} \\
+\frac{E I}{\rho A}\left[\frac{\partial^{4} \underline{v}(t ; \alpha)}{\partial x^{4}}, \frac{\partial^{4} \bar{v}(t ; \alpha)}{\partial x^{4}}\right]=\frac{F(x, t)}{\rho A}
\end{gathered}
$$

subject to fuzzy initial condition

$$
\begin{aligned}
{[\underline{v}(0 ; \alpha), \bar{v}(0 ; \alpha)] } & =\left[\underline{v}^{\prime}(0 ; \alpha), \bar{v}^{\prime}(0 ; \alpha)\right] \\
& =[0.1 \alpha-0.1,0.1-0.1 \alpha],
\end{aligned}
$$

where $\alpha \in[0,1]$.

Next, using the double parametric form (as discussed in Definition 2) above (11), it can be expressed as

$$
\begin{aligned}
& \left\{\beta\left(\frac{\partial^{2} \bar{v}(t ; \alpha)}{\partial t^{2}}-\frac{\partial^{2} \underline{v}(t ; \alpha)}{\partial t^{2}}\right)+\frac{\partial^{2} \underline{v}(t ; \alpha)}{\partial t^{2}}\right\} \\
& \quad+\frac{c}{\rho A}\left\{\beta\left(\frac{\partial \bar{v}(t ; \alpha)}{\partial t}-\frac{\partial \underline{v}(t ; \alpha)}{\partial t}\right)+\frac{\partial \underline{v}(t ; \alpha)}{\partial t}\right\} \\
& \quad+\frac{E I}{\rho A}\left\{\beta\left(\frac{\partial^{4} \bar{v}(t ; \alpha)}{\partial x^{4}}-\frac{\partial^{4} \underline{v}(t ; \alpha)}{\partial x^{4}}\right)+\frac{\partial^{4} \underline{v}(t ; \alpha)}{\partial x^{4}}\right\} \\
& =\frac{F(x, t)}{\rho A}
\end{aligned}
$$

subject to the fuzzy initial conditions

$$
\begin{aligned}
\{\beta & (\bar{v}(0 ; \alpha)-\underline{v}(0 ; \alpha))+\underline{v}(0 ; \alpha)\} \\
& =\left\{\beta\left(\bar{v}^{\prime}(0 ; \alpha)-\underline{v}^{\prime}(0 ; \alpha)\right)+\underline{v}^{\prime}(0 ; \alpha)\right\} \\
& =\{\beta(0.2-0.2 \alpha)+(0.1 \alpha-0.1)\},
\end{aligned}
$$

where $\beta \in[0,1]$.

Let us now denote

$$
\begin{gathered}
\left\{\beta\left(\frac{\partial^{2} \bar{v}(t ; \alpha)}{\partial t^{2}}-\frac{\partial^{2} \underline{v}(t ; \alpha)}{\partial t^{2}}\right)+\frac{\partial^{2} \underline{v}(t ; \alpha)}{\partial t^{2}}\right\}=\frac{\partial^{2} v(t ; \alpha, \beta)}{\partial t^{2}}, \\
\left\{\beta\left(\frac{\partial \bar{v}(t ; \alpha)}{\partial t}-\frac{\partial \underline{v}(t ; \alpha)}{\partial t}\right)+\frac{\partial \underline{v}(t ; \alpha)}{\partial t}\right\}=\frac{\partial v(t ; \alpha, \beta)}{\partial t}, \\
\left\{\beta\left(\frac{\partial^{4} \bar{v}(t ; \alpha)}{\partial x^{4}}-\frac{\partial^{4} \underline{v}(t ; \alpha)}{\partial x^{4}}\right)+\frac{\partial^{4} \underline{v}(t ; \alpha)}{\partial x^{4}}\right\}=\frac{\partial^{4} v(t ; \alpha, \beta)}{\partial x^{4}}, \\
\{\beta(\bar{v}(0 ; \alpha)-\underline{v}(0 ; \alpha))+\underline{v}(0 ; \alpha)\}=v(0 ; \alpha, \beta), \\
\left\{\beta\left(\bar{v}^{\prime}(0 ; \alpha)-\underline{v}^{\prime}(0 ; \alpha)\right)+\underline{v}^{\prime}(0 ; \alpha)\right\}=v^{\prime}(0 ; \alpha, \beta) .
\end{gathered}
$$


Substituting these values in (13), we get

$$
\frac{\partial^{2} v(t ; \alpha, \beta)}{\partial t^{2}}+\frac{c}{\rho A} \frac{\partial v(t ; \alpha, \beta)}{\partial t}+\frac{E I}{\rho A} \frac{\partial^{4} v(t ; \alpha, \beta)}{\partial x^{4}}=\frac{F(x, t)}{\rho A},
$$

with initial conditions

$$
v(0 ; \alpha, \beta)=v^{\prime}(0 ; \alpha, \beta)=\{\beta(0.2-0.2 \alpha)+(0.1 \alpha-0.1)\} .
$$

Hence, solving the corresponding crisp differential equation, one may get the solution as $v(t ; \alpha, \beta)$. To obtain the lower and upper bound of the solution in single parametric form, we may put $\beta=0$ and 1 , respectively. This may be represented as

$$
v(t ; \alpha, 0)=\underline{v}(t, \alpha), \quad v(t, \alpha, 1)=\bar{v}(t, \alpha) .
$$

Now, to solve (16), we have applied homotopy perturbation method. According to HPM, we may construct a simple homotopy for an embedding parameter $p \in[0,1]$ as follows:

$$
\begin{aligned}
(1-p) \frac{\partial^{2} v(t ; \alpha, \beta)}{\partial t^{2}}+p & {\left[\frac{\partial^{2} v(t ; \alpha, \beta)}{\partial t^{2}}+\frac{c}{\rho A} \frac{\partial v(t ; \alpha, \beta)}{\partial t}\right.} \\
& \left.+\frac{E I}{\rho A} \frac{\partial^{4} v(t ; \alpha, \beta)}{\partial x^{4}}-\frac{F(x, t)}{\rho A}\right]=0
\end{aligned}
$$

or

$$
\begin{aligned}
& \frac{\partial^{2} v(t ; \alpha, \beta)}{\partial t^{2}} \\
& \quad+p\left[\frac{c}{\rho A} \frac{\partial v(t ; \alpha, \beta)}{\partial t}+\frac{E I}{\rho A} \frac{\partial^{4} v(t ; \alpha, \beta)}{\partial x^{4}}-\frac{F(x, t)}{\rho A}\right]=0 .
\end{aligned}
$$

Here, $p$ is considered as a small homotopy parameter $0 \leq p \leq$ 1. For $p=0$, (19) and (20) become a linear equation; that is, $\partial^{2} v(t ; \alpha, \beta) / \partial t^{2}=0$, which is easy to solve. For $p=1$, (19) and (20) turn out to be same as the original equation (16). This is called deformation in topology. $\partial^{2} v(t ; \alpha, \beta) / \partial t^{2}$ and $(c / \rho A)(\partial v(t ; \alpha, \beta) / \partial t)+(E I / \rho A)\left(\partial^{4} v(t ; \alpha, \beta) / \partial x^{4}\right)-$ $(F(x, t) / \rho A)$ are called homotopic. Next, we can assume the solution of (19) or (20) as a power series expansion in $p$ as

$$
\begin{aligned}
v(t ; \alpha, \beta)= & v_{0}(t ; \alpha, \beta)+p v_{1}(t ; \alpha, \beta) \\
& +p^{2} v_{2}(t ; \alpha, \beta)+p^{3} v_{3}(t ; \alpha, \beta)+\cdots,
\end{aligned}
$$

where $v_{i}(t ; \alpha, \beta)$ for $i=0,1,2,3, \ldots$ are functions yet to be determined. Substituting (21) into (19) or (20) and equating the terms with the identical powers of $p$, we have

$$
\begin{gathered}
p^{0}: \frac{\partial^{2} v_{0}(t ; \alpha, \beta)}{\partial t^{2}}=0, \\
p^{1}: \frac{\partial^{2} v_{1}(t ; \alpha, \beta)}{\partial t^{2}}+\frac{c}{\rho A} \frac{\partial v_{0}(t ; \alpha, \beta)}{\partial t} \\
+\frac{E I}{\rho A} \frac{\partial^{4} v_{0}(t ; \alpha, \beta)}{\partial x^{4}}-\frac{F(x, t)}{\rho A}=0,
\end{gathered}
$$

$$
\begin{gathered}
p^{2}: \frac{\partial^{2} v_{2}(t ; \alpha, \beta)}{\partial t^{2}}+\frac{c}{\rho A} \frac{\partial v_{1}(t ; \alpha, \beta)}{\partial t} \\
+\frac{E I}{\rho A} \frac{\partial^{4} v_{1}(t ; \alpha, \beta)}{\partial x^{4}}=0, \\
p^{3}: \frac{\partial^{2} v_{3}(t ; \alpha, \beta)}{\partial t^{2}}+\frac{c}{\rho A} \frac{\partial v_{2}(t ; \alpha, \beta)}{\partial t} \\
+\frac{E I}{\rho A} \frac{\partial^{4} v_{2}(t ; \alpha, \beta)}{\partial x^{4}}=0,
\end{gathered}
$$

and so on.

Choosing initial approximation $v(0 ; \alpha, \beta)=v^{\prime}(0 ; \alpha, \beta)$ and applying the inverse operator $L_{t t}^{-1}$ (which is the inverse of the operator $\left.L_{t t}=\partial^{2} / \partial t^{2}\right)$ on both sides of every equation in (22) one may obtain the following equations:

$$
v_{0}(t ; \alpha, \beta)=v^{\prime}(0 ; \alpha, \beta) t+v(0 ; \alpha, \beta)
$$

$$
\begin{aligned}
& v_{1}(t ; \alpha, \beta) \\
& =L_{t t}^{-1}\left(-\frac{c}{\rho A} \frac{\partial v_{0}(t ; \alpha, \beta)}{\partial t}-\frac{E I}{\rho A} \frac{\partial^{4} v_{0}(t ; \alpha, \beta)}{\partial x^{4}}+\frac{F(x, t)}{\rho A}\right), \\
& v_{2}(t ; \alpha, \beta)=L_{t t}^{-1}\left(-\frac{c}{\rho A} \frac{\partial v_{1}(t ; \alpha, \beta)}{\partial t}-\frac{E I}{\rho A} \frac{\partial^{4} v_{1}(t ; \alpha, \beta)}{\partial x^{4}}\right), \\
& v_{3}(t ; \alpha, \beta)=L_{t t}^{-1}\left(-\frac{c}{\rho A} \frac{\partial v_{2}(t ; \alpha, \beta)}{\partial t}-\frac{E I}{\rho A} \frac{\partial^{4} v_{2}(t ; \alpha, \beta)}{\partial x^{4}}\right),
\end{aligned}
$$

and so on.

Now, substituting these terms in (21) with $p \rightarrow 1$, one may get the approximate solution of (16) as

$$
\begin{aligned}
v(t ; \alpha, \beta)= & v_{0}(t ; \alpha, \beta)+v_{1}(t ; \alpha, \beta) \\
& +v_{2}(t ; \alpha, \beta)+v_{3}(t ; \alpha, \beta)+\cdots
\end{aligned}
$$

The solution series converge very rapidly. Proof of convergence of the above series may be found in [22, 23]. The rapid convergence means that only few terms are required to get the approximate solutions.

\section{Uncertain Response Analysis}

Let us consider the external applied force $F(x, t)$ as

$$
F(x, t)=f(x) g(t),
$$

where $f(x)$ is a specified space dependent deterministic function and $g(t)$ is time dependent process. In the following paragraph, we will examine the uncertain response of the dynamic system (5) subject to two different loading conditions.

5.1. Unit Step Function Response. Now, the response of the beam subject to a unit step load has been considered of 
the form $g(t)=B u(t)$, where $u(t)$ is the Heaviside function and $B$ is a constant. By using HPM, we have

$$
\begin{aligned}
v_{0}(t ; \alpha, \beta)=\{\beta( & 0.2-0.2 \alpha)+(0.1 \alpha-0.1)\}(1+t), \\
v_{1}(t ; \alpha, \beta)= & \{\beta(0.2-0.2 \alpha)+(0.1 \alpha-0.1)\} \\
& \times\left(-\frac{c}{\rho A} \frac{t^{2}}{2}\right)+\frac{f B t^{2}}{2 \rho A}, \\
v_{2}(t ; \alpha, \beta)= & \{\beta(0.2-0.2 \alpha)+(0.1 \alpha-0.1)\} \\
& \times\left(\frac{c^{2}}{\rho^{2} A^{2}} \frac{t^{3}}{\Gamma(4)}\right) \\
& -\frac{f B c}{\rho^{2} A^{2}} \frac{t^{3}}{\Gamma(4)}-\frac{E I B f^{4}}{\rho^{2} A^{2}} \frac{t^{4}}{\Gamma(5)}, \\
v_{3}(t ; \alpha, \beta)= & \{\beta(0.2-0.2 \alpha)+(0.1 \alpha-0.1)\} \\
& \times\left(-\frac{c 3}{\rho^{3} A^{3}} \frac{t^{4}}{\Gamma(5)}\right) \\
& +\frac{f B c^{2}}{\rho^{3} A^{3}} \frac{t^{4}}{\Gamma(5)}+\frac{2 E I B c f^{4}}{\rho^{3} A^{3}} \frac{t^{5}}{\Gamma(6)} \\
& +\frac{E^{2} I^{2} B f^{8}}{\rho^{3} A^{3}} \frac{t^{6}}{\Gamma(7)},
\end{aligned}
$$

and so on, where $f^{(i)}=\partial^{i} f / \partial x^{i}$.

In a similar manner, the rest of the components can be obtained. Therefore, the solution can be written in a general form as

$$
\begin{aligned}
v(t ; \alpha, \beta)= & \{\beta(0.2-0.2 \alpha)+(0.1 \alpha-0.1)\} \\
& \times\left\{\left(1+\frac{\rho A}{c}\right)-\left(\frac{\rho A}{c} e^{(-c / \rho A) t}\right)\right\} \\
& +\frac{1}{\rho A} \sum_{r=0}^{\infty} \frac{(-1)^{r}}{r !}\left(\frac{E I}{\rho A}\right)^{r} f^{(4 r)} t^{2(r+1)} \\
& \times \sum_{j=0}^{\infty}\left(\frac{-c}{\rho A}\right)^{j} \frac{(j+r) ! t^{j}}{j ! \Gamma(j+2 r+3)} .
\end{aligned}
$$

To obtain the solution bound in single parametric form we may put $\beta=0$ and 1 , respectively, to get the lower and upper bound of the solution as

$$
\begin{aligned}
\underline{v}(t ; \alpha, 0)= & \underline{v}(t ; \alpha) \\
= & (0.1 \alpha-0.1)\left\{\left(1+\frac{\rho A}{c}\right)-\left(\frac{\rho A}{c} e^{(-c / \rho A) t}\right)\right\} \\
& +\frac{1}{\rho A} \sum_{r=0}^{\infty} \frac{(-1)^{r}}{r !}\left(\frac{E I}{\rho A}\right)^{r} f^{(4 r)} t^{2(r+1)} \\
& \times \sum_{j=0}^{\infty}\left(\frac{-c}{\rho A}\right)^{j} \frac{(j+r) ! t^{j}}{j ! \Gamma(j+2 r+3)},
\end{aligned}
$$

$$
\begin{aligned}
\bar{v}(t ; \alpha, 1)= & \bar{v}(t ; \alpha) \\
= & (0.1-0.1 \alpha) \\
& \times\left\{\left(1+\frac{\rho A}{c}\right)-\left(\frac{\rho A}{c} e^{(-c / \rho A) t}\right)\right\} \\
& +\frac{1}{\rho A} \sum_{r=0}^{\infty} \frac{(-1)^{r}}{r !}\left(\frac{E I}{\rho A}\right)^{r} f^{(4 r)} t^{2(r+1)} \\
& \times \sum_{j=0}^{\infty}\left(\frac{-c}{\rho A}\right)^{j} \frac{(j+r) ! t^{j}}{j ! \Gamma(j+2 r+3)} .
\end{aligned}
$$

5.2. Impulse Response. Next, let us consider the response of the beam subject to a unit impulse load of the form $g(t)=$ $\delta(t)$, where $\delta(t)$ is the unit impulse function. Using HPM in this case again, one may have

$$
\begin{aligned}
v_{0}(t ; \alpha, \beta)=\{\beta( & 0.2-0.2 \alpha)+(0.1 \alpha-0.1)\}(1+t) \\
v_{1}(t ; \alpha, \beta)= & \{\beta(0.2-0.2 \alpha)+(0.1 \alpha-0.1)\} \\
& \times\left(-\frac{c}{\rho A} \frac{t^{2}}{2}\right)+\frac{f t}{\rho A}, \\
v_{2}(t ; \alpha, \beta)= & \{\beta(0.2-0.2 \alpha)+(0.1 \alpha-0.1)\} \\
& \times\left(\frac{c^{2}}{\rho^{2} A^{2}} \frac{t^{3}}{\Gamma(4)}\right) \\
& -\frac{f c}{\rho^{2} A^{2}} \frac{t^{2}}{\Gamma(3)}-\frac{E I f^{4}}{\rho^{2} A^{2}} \frac{t^{3}}{\Gamma(4)} \\
v_{3}(t ; \alpha, \beta)= & \{\beta(0.2-0.2 \alpha)+(0.1 \alpha-0.1)\} \\
& \times\left(-\frac{c^{3}}{\rho^{3} A^{3}} \frac{t^{4}}{\Gamma(5)}\right) \\
& +\frac{f c^{2}}{\rho^{3} A^{3}} \frac{t^{3}}{\Gamma(4)}+\frac{2 E I c f^{4}}{\rho^{3} A^{3}} \frac{t^{4}}{\Gamma(5)} \\
& +\frac{E^{2} I^{2} f^{8}}{\rho^{3} A^{3}} \frac{t^{5}}{\Gamma(6)},
\end{aligned}
$$

and so on, where $f^{(i)}=\partial^{i} f / \partial x^{i}$.

In a similar manner, the rest of the components can be obtained. Therefore, the solution can be written in a general form as

$$
\begin{aligned}
v(t ; \alpha, \beta)= & \{\beta(0.2-0.2 \alpha)+(0.1 \alpha-0.1)\} \\
& \times\left\{\left(1+\frac{\rho A}{c}\right)-\left(\frac{\rho A}{c} e^{(-c / \rho A) t}\right)\right\} \\
& +\frac{1}{\rho A} \sum_{r=0}^{\infty} \frac{(-1)^{r}}{r !}\left(\frac{E I}{\rho A}\right)^{r} f^{(4 r)} t^{2 r+1} \\
& \times \sum_{j=0}^{\infty}\left(\frac{-c}{\rho A}\right)^{j} \frac{(j+r) ! t^{j}}{j ! \Gamma(j+2 r+2)} .
\end{aligned}
$$




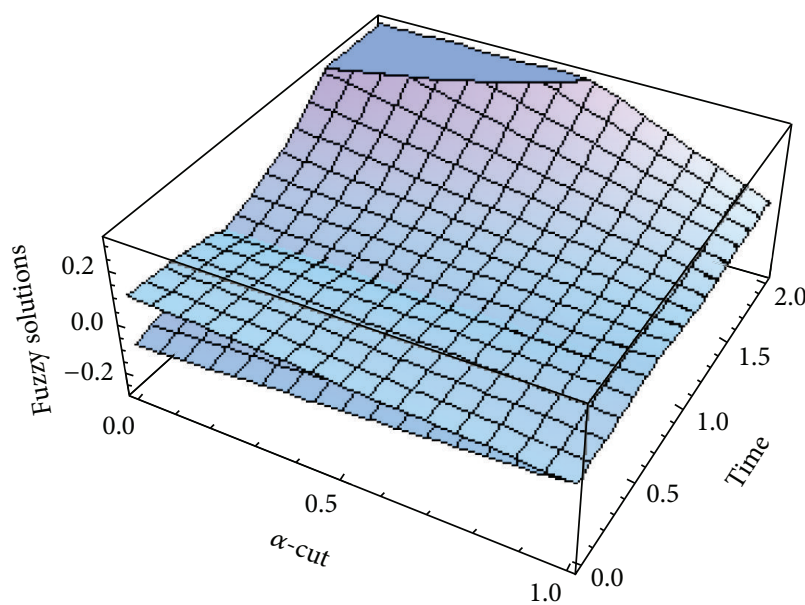

(a)

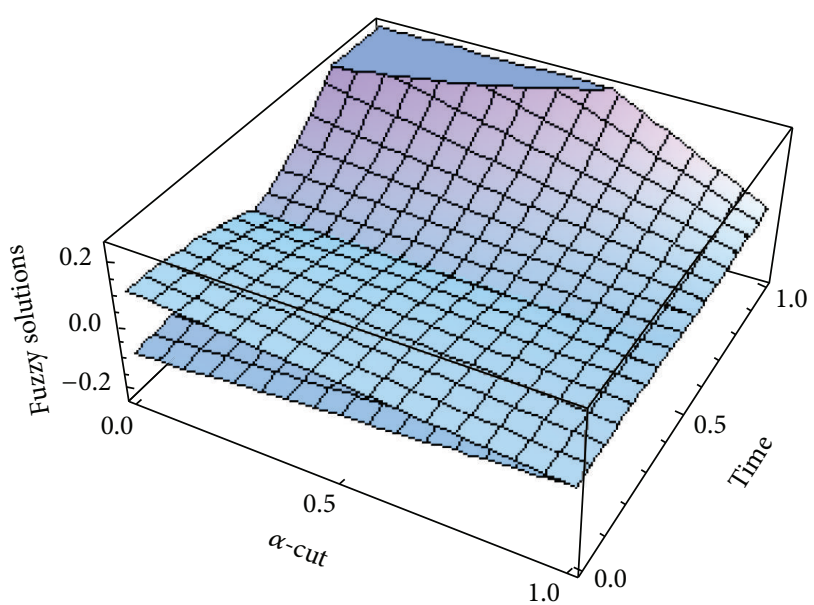

(b)

FIGURE 1: Fuzzy responses at $x=1 / 2$ with natural frequency (a) $\omega=5 \mathrm{rad} / \mathrm{s}$ and (b) $\omega=10 \mathrm{rad} / \mathrm{s}$ and damping ratio $\eta=0.05$ for unit step response.

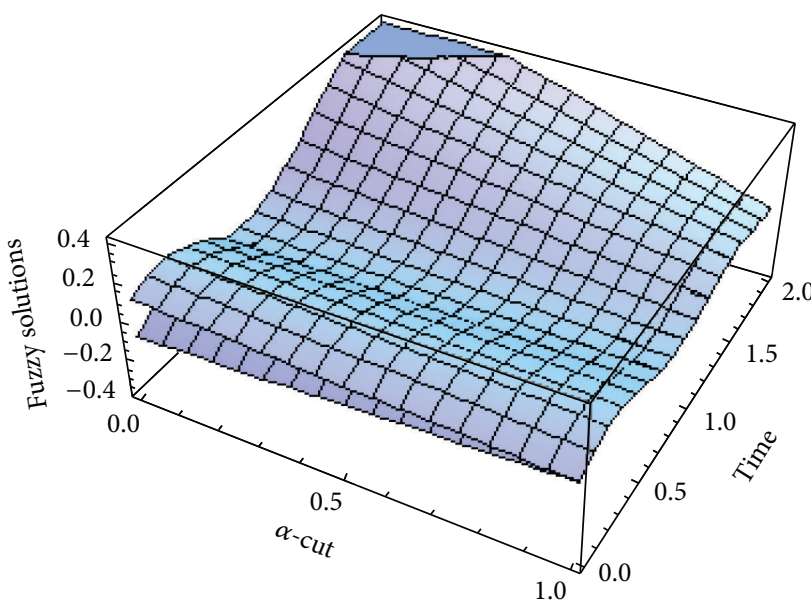

(a)

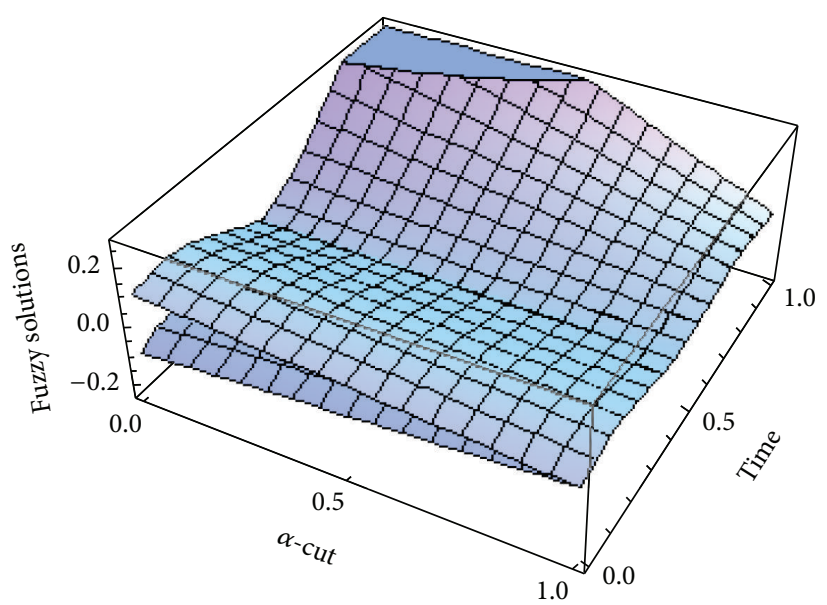

(b)

FIGURE 2: Fuzzy responses at $x=1 / 2$ with natural frequency (a) $\omega=5 \mathrm{rad} / \mathrm{s}$ and (b) $\omega=10 \mathrm{rad} / \mathrm{s}$ and damping ratio $\eta=0.05$ for unit impulse response.

Again, to obtain the solution bound for this case in single parametric form, one may put $\beta=0$ and 1 , respectively, to get the lower and upper bound of the solution as

$$
\begin{aligned}
\underline{v}(t ; \alpha, 0)= & \underline{v}(t ; \alpha) \\
= & (0.1 \alpha-0.1) \\
& \times\left\{\left(1+\frac{\rho A}{c}\right)-\left(\frac{\rho A}{c} e^{(-c / \rho A) t}\right)\right\} \\
& +\frac{1}{\rho A} \sum_{r=0}^{\infty} \frac{(-1)^{r}}{r !}\left(\frac{E I}{\rho A}\right)^{r} f^{(4 r)} t^{2 r+1} \\
& \times \sum_{j=0}^{\infty}\left(\frac{-c}{\rho A}\right)^{j} \frac{(j+r) ! t^{j}}{j ! \Gamma(j+2 r+2)},
\end{aligned}
$$

$$
\begin{aligned}
\bar{v}(t ; \alpha, 1)= & \bar{v}(t ; \alpha) \\
= & (0.1-0.1 \alpha) \\
& \times\left\{\left(1+\frac{\rho A}{c}\right)-\left(\frac{\rho A}{c} e^{(-c / \rho A) t}\right)\right\} \\
& +\frac{1}{\rho A} \sum_{r=0}^{\infty} \frac{(-1)^{r}}{r !}\left(\frac{E I}{\rho A}\right)^{r} f^{(4 r)} t^{2 r+1} \\
& \times \sum_{j=0}^{\infty}\left(\frac{-c}{\rho A}\right)^{j} \frac{(j+r) ! t^{j}}{j ! \Gamma(j+2 r+2)} .
\end{aligned}
$$

\section{Numerical Results and Discussions}

In this section, we present the numerical solution of uncertain beam equation using HPM. It is a gigantic task to include all 
the results with respect to various parameters involved in the corresponding equation here. As discussed above, uncertain responses subject to unit step and impulse responses have been considered. Computed results are depicted in terms of plots. For the numerical result, we have considered the simply supported beam and

$$
f(x)=\sin \left(\frac{\pi x}{L}\right) .
$$

Here, the numerical computations have been done by truncating the infinite series in (28) and (31) to a finite number of terms. For numerical simulations, let us denote $c / m$ and $E I / \rho A$, respectively, as $2 \eta \omega^{3 / 2}$ and $\omega^{2}$, where $\omega$ is the natural frequency and $\eta$ is the damping ratio. The values of the parameters are taken as $B=1, \rho A=1, L=\pi, x=1 / 2$, and $m=1$.

Next, by taking $x=1 / 2$ with natural frequency $\omega=$ $5 \mathrm{rad} / \mathrm{s}$ and $10 \mathrm{rad} / \mathrm{s}$ and damping ratio $\eta=0.05$ as constant and varying both the $t$ and $\alpha$, the obtained fuzzy results for unit and impulse responses are depicted in Figures 1 and 2, respectively.

As discussed above, for both cases with $\alpha=1$, the fuzzy initial condition converts into crisp initial condition. It is interesting to note that for both the responses, lower and upper bounds of the fuzzy solutions are same for $\alpha=1$.

\section{Conclusions}

In this paper, homotopy perturbation method has successfully been applied to the solution of an uncertain simply supported beam using double parametric form of fuzzy numbers. The proposed double parametric form approach is found to be easy and straightforward. Here, performance of the method is shown by using triangular fuzzy number. It is interesting to note for $\alpha=1$, in both the responses lower response is equal to the upper response. Though the solution by HPM is of the form of an infinite series, it can be written in a closed form in some cases. The main advantage of HPM is the capability to achieve exact solution and rapid convergences with few terms.

\section{Acknowledgment}

Smita Tapaswini would like to thank the UGC, Government of India, for financial support under Rajiv Gandhi National Fellowship (RGNF).

\section{References}

[1] S. L. Chang and L. A. Zadeh, "On fuzzy mapping and control," IEEE Transactions on Systems, Man and Cybernetics, vol. 2, no. 1, pp. 30-34, 1972.

[2] D. Dubois and H. Prade, "Towards fuzzy differential calculus part 3: differentiation," Fuzzy Sets and Systems, vol. 8, no. 3, pp. 225-233, 1982.

[3] O. Kaleva, "Fuzzy differential equations," Fuzzy Sets and Systems, vol. 24, no. 3, pp. 301-317, 1987.

[4] O. Kaleva, "The cauchy problem for fuzzy differential equations," Fuzzy Sets and Systems, vol. 35, no. 3, pp. 389-396, 1990.
[5] S. Seikkala, "On the fuzzy initial value problem," Fuzzy Sets and Systems, vol. 24, no. 3, pp. 319-330, 1987.

[6] M. Ma, M. Friedman, and A. Kandel, "Numerical solutions of fuzzy differential equations," Fuzzy Sets and Systems, vol. 105, no. 1, pp. 133-138, 1999.

[7] N. Mikaeilvand and S. Khakrangin, "Solving fuzzy partial differential equations by fuzzy two-dimensional differential transform method," Neural Computing and Applications, vol. 21, supplement 1, pp. 307-312, 2012.

[8] S. Abbasbandy and T. Allahviranloo, "Numerical solutions of fuzzy differential equations by taylor method," Computational Methods in Applied Mathematics, vol. 2, no. 2, pp. 113-124, 2002.

[9] S. Abbasbandy, T. Allahviranloo, Ó. López-Pouso, and J. J. Nieto, "Numerical methods forfuzzy differential inclusions," Computers and Mathematics with Applications, vol. 48, no. 10-11, pp. 1633-1641, 2004.

[10] A. Khastan, J. J. Nieto, and R. Rodríguez-López, "Variation of constant formula for first order fuzzy differential equations," Fuzzy Sets and Systems, vol. 177, no. 1, pp. 20-33, 2011.

[11] T. Allahviranloo, S. Abbasbandy, and H. Rouhparvar, "The exact solutions of fuzzy wave-like equations with variable coefficients by a variational iteration method," Applied Soft Computing Journal, vol. 11, no. 2, pp. 2186-2192, 2011.

[12] Y. Chalco-Cano and H. Román-Flores, "On new solutions of fuzzy differential equations," Chaos, Solitons and Fractals, vol. 38, no. 1, pp. 112-119, 2008.

[13] S. P. Mondal and T. K. Roy, "First order linear non homogeneous ordinary differential equation in fuzzy environment," Mathematical Theory and Modeling, vol. 3, no. 1, pp. 85-95, 2013.

[14] S. C. Palligkinis, G. Papageorgiou, and I. T. Famelis, "RungeKutta methods for fuzzy differential equations," Applied Mathematics and Computation, vol. 209, no. 1, pp. 97-105, 2009.

[15] O. Akin, T. Khaniyev, O. Oruc, and I. B. Turksen, "An algorithm for the solution of second order fuzzy initial value problems," Expert Systems with Applications, vol. 40, no. 3, pp. 953-957, 2013.

[16] S. Tapaswini and S. Chakraverty, "A new approach to fuzzy initial value problem by improved Euler method," International Journal of Fuzzy Information and Engineering, vol. 4, no. 3, pp. 293-312, 2012.

[17] T. Allahviranloo, E. Ahmady, and N. Ahmady, "Nth-order fuzzy linear differential equations," Information Sciences, vol. 178, no. 5, pp. 1309-1324, 2008.

[18] H. Jafari, M. Saeidy, and D. Baleanu, "The variational iteration method for solving $n$-th order fuzzy differential equations," Central European Journal of Physics, vol. 10, no. 1, pp. 76-85, 2012.

[19] N. Parandin, "Numerical solution of fuzzy differential equations of n-th order by Runge-Kutta method," Neural Computing \& Applications, vol. 21, supplement 1, pp. S347-S355, 2012.

[20] M. S. Hashemi, J. Malekinagad, and H. R. Marasi, "Series Solution of fuzzy differential equations," Advances in Fuzzy Systems, vol. 2012, Article ID jfsva-00066, 16 pages, 2012.

[21] Y. Zhang and G. Wang, "Time domain methods for the solutions of N-order fuzzy differential equations," Fuzzy Sets and Systems, vol. 94, no. 1, pp. 77-92, 1998.

[22] J. H. He, "Homotopy perturbation technique," Computer Methods in Applied Mechanics and Engineering, vol. 178, no. 3-4, pp. 257-262, 1999.

[23] J. H. He, "Coupling method of a homotopy technique and a perturbation technique for non-linear problems," International Journal of Non-Linear Mechanics, vol. 35, no. 1, pp. 37-43, 2000. 
[24] D. Behera and S. Chakraverty, "Numerical solution of fractionally damped beam by homotopy perturbation method," Central European Journal of Physics, 2013.

[25] S. Chakraverty and D. Behera, "Dynamic responses of fractionally damped mechanical system using homotopy perturbation method," Alexandria Engineering Journal, 2013.

[26] T. Allahviranloo and S. Hashemzehi, "The homotopy perturbation method for fuzzy Fredholm integral equations," Journal of Applied Mathematics, Islamic Azad University of Lahijan, vol. 5, pp. 1-12, 2008.

[27] E. Babolian, A. R. Vahidi, and Z. Azimzadeh, "An improvement to the homotopy perturbation method for solving integro-differential equations," International Journal of Industrial Mathematics, vol. 4, pp. 353-363, 2012.

[28] M. Matinfar and M. Saeidy, "Application of Homotopy perturbation method for fuzzy integral equations," The Journal of Mathematics and Computer Science, vol. 1, pp. 377-385, 2010.

[29] M. Ghanbari, "Numerical solution of fuzzy initial value problems under generalized differentiability by HPM," International Journal of Industrial Mathematics, vol. 1, no. 1, pp. 19-39, 2009.

[30] S. Tapaswini and S. Chakraverty, "Numerical solution of $\mathrm{n}$-th order fuzzy linear differential equations by homotopy perturbation method," International Journal of Computer Applications, vol. 64, pp. 5-10, 2013.

[31] B. Bede, "Note on 'Numerical solutions of fuzzy differential equations by predictor-corrector method"' Information Sciences, vol. 178, no. 7, pp. 1917-1922, 2008.

[32] M. Z. Ahmada, M. K. Hasanb, and B. D. Baets, "Analytical and numerical solutions of fuzzy differential equations," Information Sciences, vol. 236, pp. 156-167, 2013.

[33] J. J. Buckley and T. Feuring, "Fuzzy initial value problem for Nth-order linear differential equations," Fuzzy Sets and Systems, vol. 121, no. 2, pp. 247-255, 2001. 

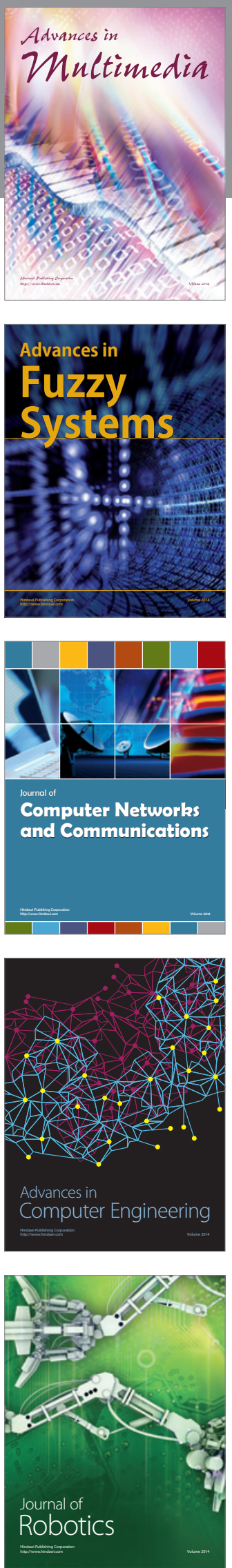

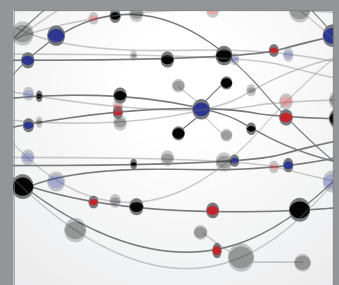

The Scientific World Journal
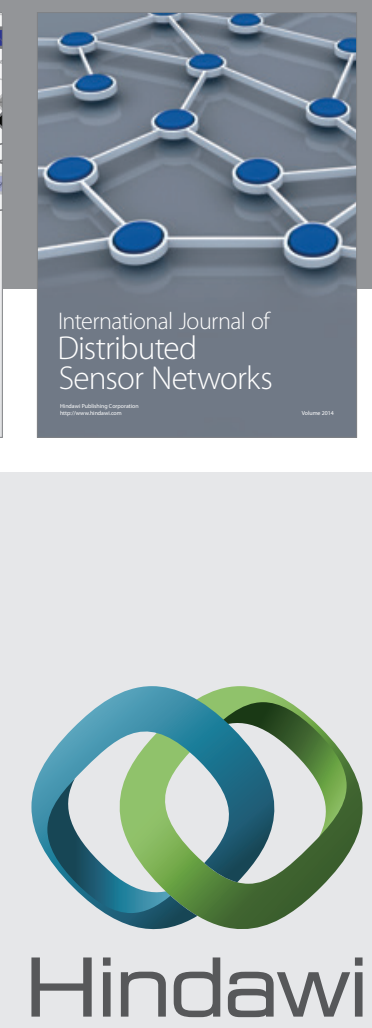

Submit your manuscripts at

http://www.hindawi.com
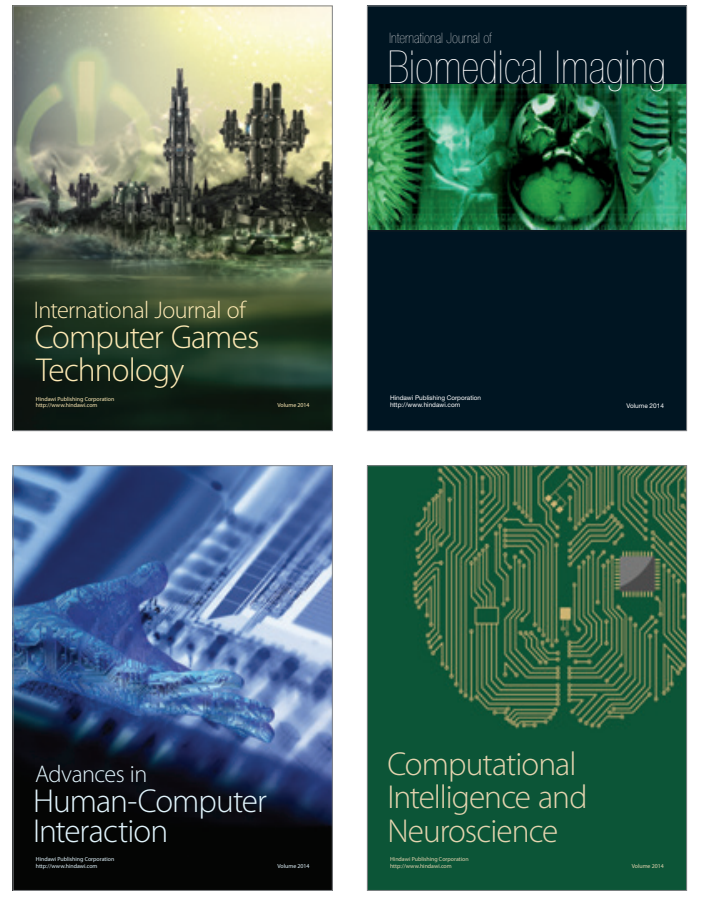
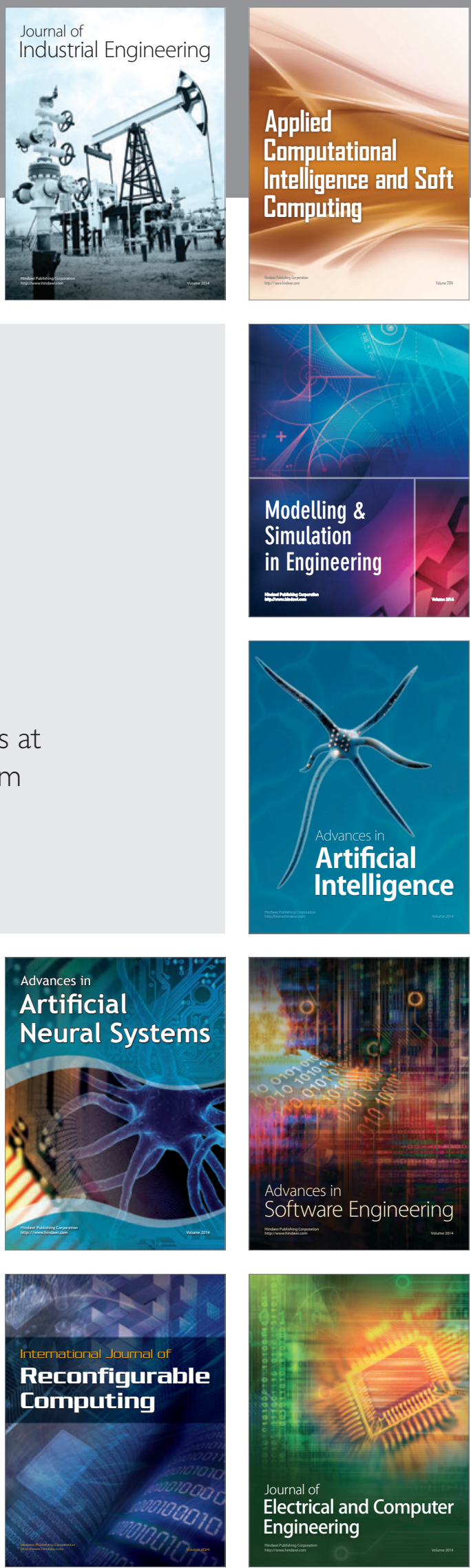